\title{
Pluriactividad del trabajo femenino: recurrencias y transiciones. Un estudio de caso en Tlaxcala, México ${ }^{1}$
}

Pluriactivity offemale work: recurrences and transitions. A case study in Tlaxcala, Mexico

\author{
JANETT VALLEJO RoMÁN \\ CIESAS-Golfo, México \\ Juan Carlos Rodríguez TORRENT \\ Universidad de Valparaíso, Chile
}

\begin{abstract}
RESUMEN El eje central de este trabajo es presentar un estudio asociado a las transformaciones experimentadas por las familias rurales y los cambios en el mercado de trabajo, enfatizando el papel actual de las mujeres en la industria textil y de confección en el municipio de Tepetitla de Lardizábal, Tlaxcala.

En el contexto de globalización neoliberal se aprecia una redirección y decantación de las mujeres al mercado del trabajo en los espacios rurales, poniendo de manifiesto: a) la necesidad de incorporación de más miembros del grupo doméstico en la búsqueda de ingresos, b) la multiplicación de sus jornadas de trabajo, c) prácticas poco justas y equitativas dentro y fuera del hogar con respecto al trabajo, y d) mayor tensión entre los miembros del hogar en el momento de la toma de decisiones respecto al trabajo fuera del hogar por parte de éstas.
\end{abstract}

PALABRAS CLAVE Mercados de trabajo, mujeres, maquiladoras y ruralidad.

ABSTRACT The central axis of this paper is to present a study associated with the transformations experienced by rural families and the labor market changes, emphasizing the current role of women in the textile and clothing industry in the municipality of Tepetitla de Lardizábal, Tlaxcala. 
In the context of neoliberal globalization there is a redirection and decantation of women to the labor market in rural areas, thus highlighting: a) the need for the incorporation of more domestic group members in the search for income, b) multiplication of women working hours, c) unfair and unequal work practices in and out of home, and d) increased tension between members of the household at the time of the decision making process related to women working outside the home.

KEYWORDS Labor markets, women, maquiladoras and rurality.

\section{Introducción}

La fase actual del capital transnacional ha llegado por presencia u omisión territorial hasta los intersticios más profundos de la vida individual y colectiva. En particular, ha trastocado los espacios rurales, modificando el territorio y las dinámicas demográficas, económicas, espaciales, sociales y culturales que le dan sentido a la existencia colectiva, es decir, las reconocidas y documentadas en los trabajos clásicos como los de Wolf $(1971,1977)$. Se trata de cambios derivados fundamentalmente por nuevos tipos de ensamblajes territoriales y campos de acción específicos nunca antes vistos, distintos a la atribución dicotómica urbano-rural que le asigna al segundo eje la condición especializada y complementaria de proveedora de alimentos para la urbe, ya que se conjugan nuevos ordenes de relaciones de poder entre lo local, lo regional, lo estatal, lo nacional y lo global.

Esta situación se ve reflejada en aspectos diversos como la disminución de la producción agrícola familiar, la necesidad de comprar lo que no se puede producir, el aumento de la migración, la diversificación ocupacional, cambios en la estructura etaria local y las relaciones de género, la entrada y salida de actores, la subcontratación, la explotación intrafamiliar, el cambio de uso del suelo, la presencia de agroindustrias, la exacerbación de la pobreza en zonas no beneficiadas por la demanda, nuevas pautas de consumo por interpenetración de la urbe y las comunicaciones, y zonas de sacrificio ambiental y cultural que terminan por definir algunas condiciones de lo que se llama la "nueva ruralidad".

Tomando estos antecedentes como punto de partida, el objetivo de este artículo es presentar un estudio de caso asociado a la transformación experimentada por las familias y las comunidades rurales en una región del Estado de Tlaxcala (4.060.923 $\mathrm{km}^{2}$ y 1.169.936 habitantes, Censo de Población 2010), y describir y analizar el nuevo papel de las "mujeres rurales" en la industria textil y maquila de confección; caracterizando los cambios y permanencias del mercado de trabajo en los espacios rurales como consecuencia de la territorialización de los nuevos ensamblajes productivos; $y$, 
analizando el rol de la mujer en la inserción en mercados amplios y su importancia en la generación de ingresos para la sobrevivencia y/o acumulación en los hogares rurales. Empíricamente se expone el caso de la industria textil y de confección, en el suroeste de Tlaxcala, particularmente en el municipio de Tepetitla de Lardizábal (véase Figura 1).

Los resultados expuestos en este artículo son producto del trabajo de campo realizado en las distintas localidades en el municipio de Tepetitla de Lardizábal. Extensos registros de campo, con entrevistas abiertas y semiestructuradas, conforman el corpus etnográfico de lo que se expone. Se realizaron cinco temporadas de trabajo de campo, en los meses de noviembre y diciembre de 2014, junio y septiembre de $2015 \mathrm{y}$ febrero de 2016. Se aplicaron 216 cuestionarios que permitieron estructurar panorama general de la localidad a nivel hogar, en éste se recuperó el número de integrantes, estructura familiar, escolaridad, actividad económica, presencia de talleres textiles y de confección, entre otros datos. Sin duda esta información cuantitativa ha sido importante para caracterizar la zona de estudios, aún cuando el objetivo principal de la aplicación del cuestionario fue que la población nos identificara formalmente (como académicos e investigadores), y que al tener contacto directo con las familias se lograra reconocer actores claves para la instrumentación de entrevistas libres y semiestructuradas ${ }^{2}$, logrando configurar un panorama más amplio de las distintas actividades y dinámicas económicas de la población. Es importante mencionar que, la gran mayoría de los talleres textiles operan de manera informal, por lo que tener acceso a éstos no fue tarea fácil. Aplicar el cuestionario nos permitió tener también acceso a los hogares.

El texto se organiza de la siguiente manera: en el primer apartado se presenta una breve discusión sobre las trasformaciones rurales en un contexto global y su incidencia en los cambios en los mercados de trabajos relacionados con la industria, como es el caso de las maquiladoras textiles y de confección. En segundo lugar, se trata de cómo inciden los cambios en la dinámica familiar desde la perspectiva de los roles y el poder. En los apartados tres y cuatro, se exponen los resultados de la investigación, enfatizando en el tema de la pluriactividad, las dinámicas, dificultades y retos laborales de las mujeres en un mercado de trabajo cada vez más precario. Finalmente, se plantean las conclusiones.

2. Los nombres de las personas entrevistadas fueron modificados para efectos de publicación. 
Figura 1. Mapa de localización

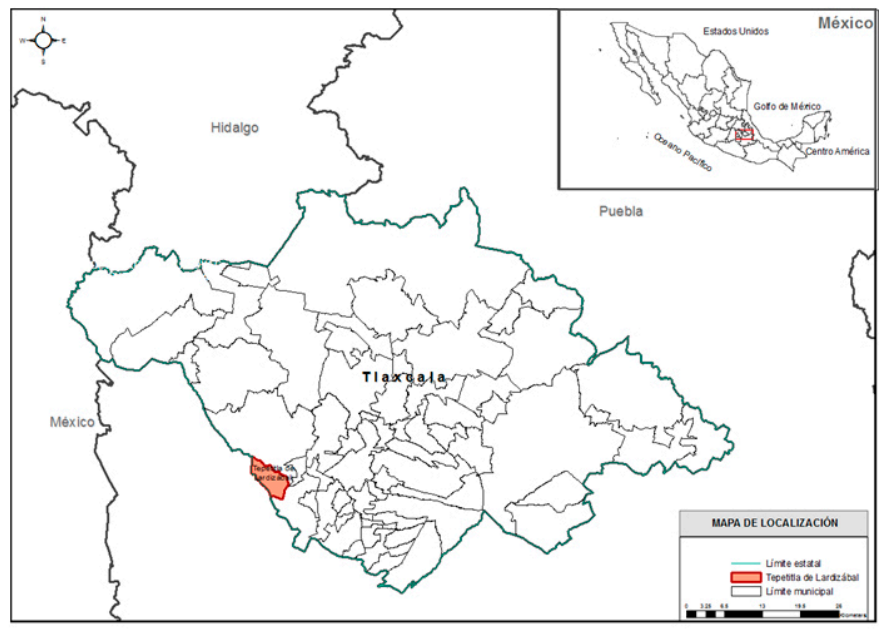

Territorio y trabajo: las ventajas competitivas de los espacios rurales en el contexto global

Se asiste a una fase del capitalismo rentista, especulativo, de localización selectiva y de cambios acelerados y profundos que afectan el funcionamiento de los estados nación o de parte de éstos, los que pueden alcanzar grandes extensiones o pequeños enclaves territoriales, así como poblaciones de distinta magnitud que experimentan las propias contradicciones del sistema capitalista (Harvey, 2014). Es la progresiva apertura y ampliación de los mercados la que produce la redistribución del trabajo y la localización del capital en territorios con mayor capacidad para atraer o generar iniciativas empresariales y/o desarrollar "emprendimientos ${ }^{3}$ ", cambiando las formas productivas y generando también eriales de pobreza y abandono. Se trata del encuentro y combinatoria de ventajas comparativas asociadas a las características de la fuerza de trabajo, las débiles leyes laborales, las infraestructuras y logística expeditas (carreteras, puertos, vías férreas) para movilizar recursos hacia los mercados de destino y favorables reglas jurídicas y económicas para la inversión.

3. Utilizamos el término emprendimiento como parte de la nomenclatura utilizada en el modelo neoliberal, ya que es usado para dar cuenta de iniciativas económicas autónomas, pero sin protección estatal y seguridad social. 
Este proceso, también llamado de deslocalización productiva, ha facilitado el desarrollo de estrategias empresariales de competencia al margen del control regulador del Estado; o, en algunos casos, con la anuencia y apoyo del mismo con cuestiones como flexibilización laboral o leyes de protección a la inversión nacional y extranjera, sin resguardo de cuestiones sanitarias y laborales para la población y de afectación ambiental a los ecosistemas. Esto ha provocado la aparición de nuevas áreas de producción desconocidas localmente, las que han incorporado a decenas de millones de nuevos trabajadores en países de tradición agrícola a formatos industriales de subcontratación o agroindustriales. Hombres, mujeres y niños, en condiciones excepcionalmente favorables para las empresas han sido desplazados de actividades tradicionales a este nuevo orden laboral para la obtención de ganancias y ampliación del capital, sin responsabilidad social y ambiental. Además, la deslocalización (o relocalización) de trabajos urbanos ha generado una fragmentación espacial, con regiones ganadoras y perdedoras; constantemente se comprueba la aparición de nuevos nodos de desarrollo o regiones productivas, a la par de la expansión o la decadencia de otras, lo que tiene efectos relevantes en la vida cotidiana producto de nuevos roles, fenómenos migratorios, desplazamiento de la fuerza de trabajo, interpenetración urbana-rural y concentración urbana.

Uno de los efectos colaterales del proceso, es lo que se conoce en economía como la "conmutación": se produce o se genera el ingreso en un lugar, pero se gasta en otro; se contamina y deterioran los ecosistemas en un lugar, y los beneficios se multiplican exponencialmente a cientos y miles de kilómetros (i.e. minería, hidrocarburos, soja, industria textil). También se observa la migración de los proveedores hacia trabajos estacionales para la reproducción de la unidad doméstica, que aún bajo condiciones salariales atractivas implica procesos de explotación indirecta de algunos de los miembros de la unidad familiar que permanecen en la comunidad (i.e. migración a Estados unidos). Si bien el trabajador provee o sostiene la reproducción cotidiana, la generacional se da a través de distintas formas de subempleo no remunerado de algunos de sus miembros (cuidado de hermanos menores y adultos en edad posproductiva), generando un trabajo extensivo e indirecto que -como en este caso de estudio- opera al servicio de la maquiladora que contrata al proveedor.

A pesar de que la lógica estructural del capitalismo en la era global carece de una localización específica, debido a los flujos financieros de capital, esto no significa que sea a-espacial, es decir, que no tenga una base territorial. Por el contrario, el capital financiero y especulativo requiere de un lugar de operación y de localización, o "glocalizado" en los términos de Robertson (2003). El capital no se reproduce en abstracto, necesita anclajes; esto es: andamios geográficos socialmente producidos y reproducidos sobre, en, y a través de los cuales las diferentes formas del capital son sucesivamente des-y re-territorializadas (Brenner, 1999: 434; Sassen, 2015). Aunque el capital 
es cada vez más móvil, la fuerza de trabajo se concentra, deprime y rearticula a través de la conjugación de factores de expulsión, retención y de atracción, así como de la infraestructura y el desarrollo de cuestiones logísticas que facilitan el establecimiento en un lugar y territorio concreto.

La fragmentación de los procesos de producción asociada a los paradigmas de flexibilidad, han favorecido la dispersión territorial de las empresas de distintos rubros y sus procesos, en lugares con bases productivas asociadas a memorias laborales especializadas como el trabajo agrícola directo y con fuerza de trabajo familiar. De este modo, aparecen en el territorio mexicano las maquiladoras como expresión de interpenetración de tareas industriales urbanas en zonas agrícolas como las que describiremos. Estas empresas maquiladoras pueden ser definidas, en términos generales, en una doble acepción: la primera, referida a aquellas plantas ensambladoras que las compañías extranjeras o nacionales instalan en determinados lugares (como en el Estado de Tlaxcala) con el objetivo de aprovechar las ventajas comparativas y competitivas que se ofrecen localmente (i.e. fuerza de trabajo disponible y disciplinable), propiciando el traslado de algunas de las actividades productivas hacia lugares con otras memorias productivas. Especialmente, se trata del traslado de aquellas que demandan mayor uso de mano de obra a los países en desarrollo o zonas económicamente deprimidas, asegurando la mayor rentabilidad por el abaratamiento de la mano de obra y de los costos generales de producción ${ }^{4}$. La segunda acepción, consiste en entender a las maquiladoras a través del fortalecimiento de talleres de escala menor, especializados, y donde se genera uno o varios de los procesos que requiere la producción de una prenda de vestir. Esta variante aplica para talleres familiares con enlaces consanguíneos o políticos, y ha sido impulsada por la reestructuración productiva e intensificadas por las empresas industriales transnacionales y también nacionales ${ }^{5}$, para enfrentar la crisis de largo plazo del capitalismo. Así, éstas unidades productivas pensadas en red o como cluster aparecen como un eslabón significativo en el volumen productivo de la cadena industrial de la empresa global, la que glocalmente alcanza a la unidad doméstica al sustraer a algunos de sus miembros de las labores agrícolas tradicionales y como manifestación de una propiedad estratégica para alcanzar umbrales más altos de acumulación.

4. Esta condición es posible porque las legislaciones son menos exigentes, existe un déficit de regulación y una baja cobertura del estado-nación.

5. Recuérdese que México posee un mercado interno suficientemente amplio, de casi 120.000.000, y 350.000.000 en Estados Unidos. 
Los talleres textiles son autónomos, operan de acuerdo a su capacidad productiva, sus propias autoexigencias y disponibilidad para delegar responsabilidades familiares de los adultos sobre niños y ancianos en otros miembros de la unidad familiar. En este sentido, es importante mencionar que detallar tipológicamente la diversidad de talleres de confección que registramos en la localidad implicaría un artículo en sí mismo. Sin embargo, podemos decir que en el municipio de Tepetitla de Lardizábal existen tantos talleres como fases específicas en la elaboración del pantalón de mezclilla.

A saber: diseño, corte, ensamblado, lavado (lijado, pigmentación y despigmentación) y terminados (elaboración de ojal, planchado, deshilado, aplicaciones de pedrería, bordados, estampado y etiquetado).

De la misma forma en que existe una diversidad especializada en cuanto a la actividad específica que se realiza en cada taller, también se presenta una desigual variedad en cuanto al tamaño en términos de espacio, infraestructura, personal ocupado en las labores y las formas de operación. Por ejemplo, los talleres llamados "medianos", con mayor número de maquinaría y de personal subcontratado, requieren de un espacio con cierta holgura para su operación; mientras que los talleres "unipersonales", donde solo cuentan con una máquina, permite trabajar en el domicilio. Para las mujeres, quienes dominan el rubro, y sobre todo las que tienen hijos, subjetivamente esta segunda opción la consideran la más adecuada; sostienen que al estar en casa les permite un mayor control y continuar con el cuidado de sus hijos, con atención a las múltiples tareas del hogar, generando menor tensión en su relación de pareja, aunque esto implica una mayor carga de trabajo. Como lo menciona Marlen:

Cuando trabajas en casa se te duplica o triplica el trabajo. Durante diez años trabajé maquilando pretina y presilla, era todo lo que hacía. Ya después me mandaban cortes completos, tenía que unir delantero, trasero, bolsa, pretina...todo. Muy pesado (...) me levantaba a las 4:30, hacer el almuerzo para mi esposo (...) después preparaba a mis tres niños, uno pal (sic) kinder y dos a la primaria, llevarlos a la escuela. De ahí pasaba por mi corte, me lo traía en la espalda, de ahí a coser sin parar hasta la una. De ahí ve al mercado y has la comida, ve por los niños, dales de comer, medio arreglas la casa y otra vez a coser hasta las nueve. Dar de cenar y bañar a los niños. Lavar, planchar y todo lo que no hiciste en el día. Ya para dormirme a las 12 de la noche (...) y mientras coses y tienes hijos, tienes que atenderlos, no es lo mismo que si trabajas en un taller. Ahí sí te concentras y sacas más rápido el trabajo (...) en casa estás como dicen: un ojo al gato y otro al garabato (A. Marlen, comunicación personal, 18 febrero de 2015).

El caso de estudio es un ejemplo claro del fomento de la tercerización industrial o modelo maquilador (Alonso, 2002), ya que la demanda no es el mercado local. El ta- 
ller permite observar el proceso económico global de ensamblaje y la deslocalización productiva, y el surgimiento de las más diversas formas y cadenas de subcontratación intra y extrafamiliar; y, simultáneamente de reescritura territorial de una zona de base agrícola que opera complementariamente en la economía del hogar.

La complejidad del caso se hace evidente por la diversidad de actores y relaciones que integran la cadena de producción: empresa matriz-empresa subcontratada; empresa subcontratada-empresa subcontratada, empresa subcontratada-talleres domésticos, talleres doméstico-taller doméstico. Es decir, existe una fragmentación y diversidad operativa al interior de la cadena productiva que desde la perspectiva de la demanda es altamente eficiente: trabajadores sin contrato, sin licencias médicas, sin leyes laborales, sin organizaciones. Cuestiones subyacentes que permiten maximizar la eficiencia de la producción de escala de prendas de vestir; donde se generan rentabilidades distintas en cada momento y diversas formas de sujeción en el proceso, así como explotación y autoexplotación familiar que finalmente redefinen el mercado de trabajo, los territorios rurales y la importancia y lugar de la producción agrícola ${ }^{6}$.

En el caso de las maquiladoras de Tlaxcala, especializadas en la confección de jeans, lo importante es que esta fragmentación de la producción promovida por las grandes empresas es adoptada, adaptada y reproducida por medianos y pequeños talleres que operan para la producción local, regional y nacional. No decimos mundial, porque el volumen del mercado mexicano es lo suficientemente atractivo para cualquier empresa.

Así, este modelo especializado por unidades productivas autónomas, ha sido apropiado y naturalizado por los pobladores como una medida eficiente para hacer frente a la crisis de la pequeña producción agrícola (agotamiento, baja productividad e insuficiencia de tierra) y de la industria textil tradicional tlaxcalteca (antiguas hilanderías). Encontrando en los talleres de confección encadenados, por una parte, una nueva forma de operar de la industria y de la confección a bajo costo; y, por otra, fortaleciendo una alternativa de ingresos y sobrevivencia para familias con memoria en la agricultura familiar.

La instalación de maquiladoras en sus distintas especializaciones y escalas, obedece -entre otras cosas- al impulso gubernamental para el desarrollo de actividades industriales en los espacios rurales y periurbanos. El presupuesto subyacente es que, en estas zonas agrícolamente deprimidas, existen ventajas competitivas (y también necesidades) como la disposición de espacio susceptible de ser redefinido o transformado en suelo (cambio de uso de tierras agrícolas), disponibilidad de mano de obra, adecuada localización geográfica con fines de mercado (distancia y fletes) e infraestructura física para los procesos.

6. Trabajar en un taller en cualquiera de los encadenamientos o especialidades, no implica que dejen de autodefinirse también como campesinos o trabajadores rurales, que el campo no esté presente en sus vidas, que constituya un refugio frente a adversidades o que se pueda mantener una milpa. 
Todas condiciones idóneas para la ejecución de políticas públicas en favor de la “industrialización", con lo cual se detonaría el “desarrollo económico”. En México, este proceso de estímulo y de interpenetración urbano-rural, solo es comprensible por deterioro de las actividades agrícolas del campo mexicano (Véase Arias, 2013; Martínez, 2015; Salas \& Rivermar, 2014).

El estímulo de políticas públicas en favor de la industria en los poblados rurales ha sido presentado también como un símbolo de modernidad. Ahora, se le muestra como un grado superior en el mundo del trabajo, bajo un discurso de competitividad y emprendimiento exitoso, lo que ha agudizado las condiciones de pobreza y disminuido la capacidad productiva de los agricultores medianos y pequeños, ya que los apoyos dirigidos al desarrollo rural no están orientados a la producción agrícola sino a la reconversión hacia otros sectores de la economía como la industria, comercio y servicios personales.

Las consecuencias no han sido del todo halagüeñas. Una buena parte de la población que anteriormente dependía de la actividad agrícola como fuente principal de ingresos, no siempre está "lista" para involucrarse en otro tipo de actividad, o para instalar un negocio propio, ya que no cuentan con las competencias y/o habilidades necesarias. Conforme a lo señalado, la única alternativa para su sobrevivencia ha sido la venta de su fuerza de trabajo en los nuevos mercados laborales que funcionan formalmente por oposición a la actividad agrícola -aunque sin acabar con ella-, con actividades rutinarias, mecánicas y de aprendizaje rápido que tienden a la especialización en la cadena textil y de confección, bajo condiciones precarias ${ }^{7}$ y sin protección en la mayoría de los casos.

Estos cambios de orientación también han modificado cuantitativa y cualitativamente el trabajo femenino. Por ejemplo, Beneria (2006), desde la economía feminista muestra cómo las mujeres contribuyen al proceso del desarrollo económico, señalando que su incorporación al mercado laboral ha ido acompañada de una serie de contradicciones y paradojas en el desenvolvimiento no sólo de las economías emergentes. Existen ciertos sectores en los que las mujeres se emplean y en los cuales en muchas ocasiones ganan autonomía, aunque también pierden en otras dimensiones. Por ejemplo, visualiza que la generación de ingresos propios corre en paralelo con su "obligada" jornada de trabajo en el hogar; o, en su defecto, a la explotación indirecta de hijos mayores que cubren aquello no remunerado por la industria, en labores de crianza de sus hermanos o en el cuidado de personas en edad posproductiva.

Se ha abierto una importante oferta laboral remunerada para las mujeres en este municipio. Pero, si bien es cierto existe una valoración positiva en cuanto a la importancia de recibir un salario por su trabajo, los empleos a los que en su mayoría tienen acceso son repetitivos, precarios, flexibles y temporales, con pocas o nulas prestaciones, jornadas exhaustivas, con límites conforme a la necesidad y urgencia, y pocas

7. Un espacio individualizado como taller, puede ser simultáneamente un lugar para comer o ver televisión. 
oportunidades de desarrollo personal. Por ello, lo importante es cómo leemos el dato estadístico del incremento de la fuerza laboral femenina en México, que se vuelve un elemento tan socorrido cuando se trata de dar cuenta pública de la composición de la fuerza de trabajo en una región o en el país.

En este tenor, Guadarrama ofrece un análisis interesante de la incorporación de las mujeres al mercado de trabajo:

(...) la incorporación de las mujeres al mercado de trabajo creció considerablemente en este contexto de apertura y flexibilización de las relaciones laborales. (...) La discusión sobre las formas de inserción femenina en el mercado de trabajo, implica reconocer los estereotipos sociales incubados desde la familia y la escuela y reproducidos en el mercado del trabajo, es también parte de una discusión sobre las formas de trabajo flexibles feminizadas que profundizan las desigualdades en la división sexual del trabajo doméstico y extradoméstico. Una prueba de ello es que el trabajo femenino se ha concentrado en los servicios de menor paga, lo cual permite también que las mujeres continúen tomando la mayor responsabilidad del trabajo doméstico. En otras palabras, el trabajo flexible en su forma más pura, como empleo fluido y temporal, excluyente, vulnerable, con riesgos, es el trabajo ejecutado por las mujeres (Guadarrama, 2008: 336).

Con lo anterior, se puede sostener que el mercado del trabajo al que se alude está conformado tanto por variables económicas producto de la globalización neoliberal, el deterioro de la pequeña producción agrícola, políticas laborales y económicas propuestas y ejecutadas por los distintos niveles de gobierno, pero también está permeado por las normas sociales y culturales propias de las regiones y poblaciones.

\section{Mercados de trabajo y dinámica familiar}

Los actuales estudios sobre el mercado del trabajo sugieren el reconocimiento de dimensiones transversales como la familia, el género, la educación, las políticas públicas, el consumo, el individuo y sus expectativas; ya que todas, en particular o enlazadas, intervienen en la formación y transformación de los mercados del trabajo. No se puede perder de vista el mercado entendido como el espacio de compra-venta y oferta-demanda de fuerza del trabajo, pero éste se debe comprender como parte de procesos sociales y culturales deslocalizados, más amplios y complejos que los que se puede apreciar en la evidencia local.

La recuperación del espacio ligado con el ámbito laboral también es referida en estudios sociológicos y antropológicos, analizando la estructura social y la memoria económica del "lugar" en donde se localiza el mercado del trabajo. Estas perspectivas 
abren un horizonte que ayuda a comprender la permanencia y transformación de las estructuras de roles y las formas institucionales. Los mercados del trabajo reflejan la sociedad de la que son parte y sus transformaciones, es decir, los procesos modernizadores locales y regionales que operan como espejos y ámbitos de imaginación del futuro y posibilidades de consumo. Así como las sociedades son producto de su historia y tradiciones culturales, también lo son los mercados del trabajo (Rau, 2006: 10); $y$, si la tierra se convierte en suelo sujeto a otros fines no productivos, los trabajadores libres se convierten en asalariados, o pasan de trabajadores libres a emprendedores que dependen de sí mismos. En este sentido, tomando en cuenta el desplazamiento del eje del saber y el hacer desde el trabajo directo en la tierra a actividades industriales desarrolladas en estos talleres, y la participación de las mujeres en el proceso, observamos en el ámbito rural un "cambio tectónico" en términos de Harvey (2014:12).

Entonces, dada la complejidad espectral de actividades, roles y nuevas aspiraciones surgidas en el proceso de reconversión laboral de lo agrícola en industrial, y la imposibilidad de reducir weberianamente a tipos ideales la adyacencia de lo rural con otras actividades laborales, resulta pertinente recurrir al termino embedednes para el análisis el cual expresa el inevitable soporte social de las acciones y las estructuras económicas, y la noción de una integración entre lo económico y lo social con un carácter no removible, salvo para fines analíticos (Gómez, 2004:161).

En la idea de embedednes, el funcionamiento de las redes sociales de reclutamiento, asignación, remuneración y movilidad están en el centro del estudio del mercado de trabajo. Bajo este argumento, los mercados de trabajo carecen de un funcionamiento autónomo e independiente a la sociedad en su conjunto, más bien se hallan inmersos en la sociedad; a tal punto que, el mercado de trabajo debe ser considerado como una institución social. Esto, debido a que en su creación y funcionamiento se generan normas, luchas, deseos y la idea de justicia, que son algunos de los elementos que trascienden las variables meramente económicas (Solow, 1992). Es decir, se pueden apreciar nuevas orientaciones y subjetividades en la configuración del enclave especializado.

El reconocimiento de estos elementos institucionalizados en el mercado de trabajo supone un tipo de sujeto racional, pero no en el sentido neoclásico, ya que su acción se puede ver influida por elementos como los de carácter afectivo, de reciprocidad, conocimiento mutuo, o de lealtad hacia quién te recluta en un taller. Recuperando la propuesta de Herrera (2005: 70), la idea de un sujeto puede ser leída como "maximizador, pero no necesariamente en relación a una ganancia puramente económica, sino que "ésta puede ser también social o simbólica. Puede tratarse es un sujeto que no está determinado por las estructuras, sino que es capaz de actuar dentro de ciertos márgenes de libertad y constreñimiento" (Herrera, 2005: 70). 
En este nivel de interacciones múltiples y recurrentes en el tiempo, para el ingreso al mercado de trabajo no sólo se fijan por medios formales. Si bien en algunos espacios laborales predominan los mecanismos formales, institucionales, legales y burocráticos, en muchos prevalecen los informales, particularistas o institucionalizados por la práctica y el conocimiento mutuo, pero no por la ley. Y, en algunos otros, existe una mezcla de formalidad e informalidad, teñido con elementos como la cercanía consanguínea o política, es decir, se configuran a partir de familias extendidas. Además, los movimientos y las posiciones laborales inciden no sólo en el salario y las capacidades del trabajador, porque también juegan un papel importante los acuerdos personales, las relaciones de confianza basadas en el parentesco, amistad, recomendaciones, personalidad y vecindad, así como las concesiones personales mutuas.

Bajo esta lógica, en el proceso de búsqueda de empleo y obtención del mismo en las maquiladoras intervienen otras variables intrafamiliares además de la económica. Los acuerdos o desacuerdos familiares de autorización son una variable a considerar para el estudio de caso. La decisión de las mujeres de emplearse muchas veces está mediada por la edad, por la posición y condición que ocupan dentro del hogar: jefa de familia, madre, hija, nuera; con o sin pareja, viuda, separada.

Como se ha reiterado, la incorporación al mercado de trabajo no sólo depende de la necesidad de obtención de ingresos para la sobrevivencia. Si bien es una de las principales motivaciones, no necesariamente la necesidad de ingresos es una variable que favorece en automático la incorporación de las mujeres al trabajo fuera del hogar. La búsqueda de trabajo para las mujeres, principalmente "fuera de casa" (i.e. en un taller medio), en la mayoría de los casos es un tema que tiene que ser "puesto a consideración": con la pareja, los padres, la suegra, o incluso con los hijos. El tipo de trabajo, el lugar de trabajo, los horarios, son muchas veces "negociados", y no siempre las favorecen, como se expondrá más adelante.

\section{Industria textil y de confección: contexto estatal y estudio de caso}

Este trabajo tiene como referente la región del suroeste de Tlaxcala, concretamente el municipio de Tepetitla de Lardizábal. Aquí existe una importante presencia industrial, la cual se ha acentuado en los últimos años, debido a las políticas públicas en favor de la industrialización y desarrollo urbano por sobre las actividades agrícola familiares. Particularmente, la industria textil tiene larga tradición en Tlaxcala. Como señala Montiel en un esfuerzo de periodización: hasta la década de los 60 del siglo XX se tenía registro de viejas fábricas dedicadas al hilado, pero fue "entre los años de 1975 y 1980 que se instalan alrededor de 250 empresas.

Se trataba de industrias nuevas y modernas que producían no sólo para el mercado nacional, sino que también exportan al mercado mundial. (...) 
Los parques, los corredores y las ciudades industriales están cambiando de manera definitiva la fase agraria y rural de la entidad (Montiel, 2009:89).

La industria textil sigue teniendo una importante presencia formal y en el imaginario en Tlaxcala. Sin embargo, el nuevo proceso de industrialización ha generado el uso intensivo de la mano de obra local. Coherente con ello, en 1978 se inauguró el camino Santa Ana Chiautempan-Puebla; y, sobre esta nueva ruta se establecieron empresas comercializadoras de diversos rubros: de aparatos electrodomésticos, loseta cerámica, piezas para la industria automotriz, alimentos balanceados para animales, fábricas de calzado y textiles. También se inauguraron fábricas en la ruta -convertida en autopista- San Martín Texmelucan-Tlaxcala, en la ciudad de Tlaxcala y en los alrededores de Apizaco. De la misma forma, encontramos el proceso en la autopista México-Puebla, inaugurada a principios de los años sesenta, lo que facilitó el traslado al Distrito Federal y reforzó aún más la hegemonía económica de la capital (Montiel, 2009: 90).

El proceso de industrialización de la región tlaxcalteca no sólo ha obedecido a factores endógenos, también la urbanización e industrialización de los municipios vecinos del Estado de Puebla han sido un factor clave para su transformación. Desde 1970 en adelante, se consolidaron nuevos polos industriales en el valle Tlaxcala-Puebla, en donde la producción y confección de prendas de mezclilla se vuelve de gran relevancia, dejando atrás los municipios industrializados tradicionales como Atlixco (industria del algodón), Teziutlán (industria metalúrgica), Tehuacán (industria de bebidas y comienzos de industria de confección), Chietla (industria de transformación de azúcar), San Pedro Cholula (industria metalúrgica y textil) y la misma ciudad de Tlaxcala (Velasco, 2014: 168).

Para el caso de estudio, el proceso de industrialización se ha agudizado con la ubicación del corredor industrial Ixtacuixtla, el cual tiene presencia directa en los municipios de Tepetitla e Ixtacuixtla, aunque su influencia de atracción de mano de obra abarca también el municipio de Nativitas. De acuerdo al Directorio Industrial del Estado de Tlaxcala, en el parque industrial Ixtacuixtla se ubican 13 empresas que operan en las siguientes ramas: alimentos y bebidas, petroquímica, textil, farmacéutica, autopartes y minerales no metálicos (Gobierno de Tlaxcala, 2014) .

Para el año 2015, el Directorio Estadístico Nacional de Unidades Económicas (DENUE) registró para el caso del Municipio de Tepetitla, 43 empresas dedicadas a la industria textil y de la confección, de las cuales 34 se dedicaban a la confección de prendas de vestir. Es importante mencionar que, oficialmente no se tiene registro de ninguna lavandería que son parte insustituible del proceso, pese a que en el municipio éstas tienen una presencia económica muy importante y de alto impacto ambiental por ser de las más contaminantes del aire y los afluentes. En el trabajo de campo se logró constatar la presencia de numerosos talleres de confección de prendas de 
mezclilla así como de lavanderías que operan de manera informal principalmente en la localidad de San Mateo Ayecac. De acuerdo a la información que arrojó nuestro instrumento estandarizado, un poco más del $60 \%$ de los hogares se registra la presencia de alguna actividad relacionada con la industria textil o de la confección, ya sea en algún taller con maquinaría sofisticada, en el trabajo a domicilio con una simple máquina recta de coser, en los terminados con una planchadora, en la elaboración manual de plantillas para decoración de pantalón, o en otra etapa del proceso de diseño, confección y terminado del pantalón de mezclilla. Este porcentaje se eleva si se considera el trabajo en la maquila que no es pagado, como el que realizan los ancianos y niños, entre los que se cuentan el deshilado, acomodar cortes y piezas, etiquetado y otras labores poco especializadas o que no requieren de la operación de maquinaría.

Otro aspecto interesante es que en el trabajo de campo se pudo corroborar la existencia de segmentación laboral por sexo en las maquiladoras. Como señala De la O:

Existe una articulación entre la expansión de la maquiladora, la reorganización de la fuerza de trabajo local y la búsqueda de suministro laboral con características específicas, como lo es el trabajo femenino. Algunos elementos indicativos de este proceso son la segmentación y polarización de ocupaciones y salarios (...) según sexo (De la $O$, 2006:93).

\section{Las mujeres en el actual esquema de la pluriactividad rural: el trabajo en la ma- quila textil y de confección}

No es reciente la discusión sobre la dependencia cada vez más común de ingresos producto de actividades no agrícolas en los espacios rurales. Además, del jefe o jefa de familia, existe la evidencia de la incorporación de otros miembros de la unidad familiar a la esfera laboral a corta edad y sin diferencia de sexo. De manera tal, que se observan cada vez más unidades familiares pluriactivas, entendiendo la pluriactividad como un proceso socioeconómico que implica la combinación de dos o más actividades generadoras de ingresos, una de las cuales puede ser la agricultura que desarrollan uno o más miembros de la unidad familiar rural. Estas actividades se pueden realizar dentro o fuera del ámbito propiamente rural (Arias, 2009; Carton de Grammont, 2008; Gras, 2004; Schneider \& Conterato, 2006).

Hoy, los factores que inciden en el reconocimiento y desarrollo de la pluriactividad son diversos. En un nivel más general se pueden mencionar aquellos elementos que influyen en las transformaciones territoriales y económicas, entre las cuales destaca el cambio en la relación rural-urbana como consecuencia de la reestructuración laboral y relocalización de actividades que eran eminentemente urbanas, teniendo claras consecuencias en la generación de nuevas alternativas de trabajo en el medio rural. La relación rural-urbana se redefinió principalmente por la cercanía de los polos de la 
agricultura familiar campesina a las ciudades, el incremento de vías de comunicación y medios de transporte que ampliaron los horizontes de mercado y consumo.

Paralelamente, se deben anexar aquellos elementos que aluden a las transformaciones en la dinámica social y económica propios de los hogares rurales. Por ejemplo, la pérdida de rentabilidad de la actividad agropecuaria por parte de los pequeños productores, aunado a la persistencia, y en algunos casos, al incremento de los niveles de pobreza; la transición de una economía basada en el equilibrio entre lo que producían y consumían, a una economía en donde el ingreso monetario regular ha cobrado mayor importancia, junto a las nuevas pautas de consumo y demandas asociadas a la educación de los hijos (Arias, 2009: 296-297 y Gras, 2004: 102-103). En un nivel más individual, asistimos a un cambio en las expectativas generacionales comúnmente asociadas a los mayores niveles de escolaridad, donde muchos jóvenes prefieren emplearse en actividades no agrícolas, ya que el campo no solo lo consideran poco rentable sino que les es ajeno (Méndez, 2008) y lo perciben como poco moderno dentro de sus expectativas. También, y de modo estructurante, el cambio y avance formal en las relaciones de género y poder, y con ello el aumento de las mujeres en el mercado de trabajo que negocian en la unidad doméstica, con nuevas expectativas y deseos de consumo.

Por cierto, es importante mencionar que el incremento de las mujeres participantes en el mercado de trabajo no es privativo de los espacios rurales. El proceso de apertura de fronteras, flexibilización y deslocalización de los procesos productivos en la fase del capitalismo neoliberal ha marcado un parteaguas en la apertura del mercado laboral femenino. Así, para el caso de México, el Instituto Nacional de Estadística e Informática (INEGI) reporta que a nivel nacional la tasa de participación en el trabajo remunerado de los hombres de 14 y más años supera significativamente (68.2\%) a la relativa a las mujeres (37.4\%). No obstante, de los 4.4 .8 millones de mujeres de 14 años y más, el 98\% realizan algún tipo de trabajo (para el mercado, o no remunerado para los hogares); mientras que para los 40.5 millones de hombres el porcentaje es de $94.2 \%$. También señala que la participación en el trabajo total de las mujeres es superior al de los hombres en todos los niveles de escolaridad. Analizando el promedio de horas que le dedican las personas ocupadas (empleadas) al trabajo total por grupo de edad, se observa que en todos los rubros las mujeres dedican más horas que los hombres, es decir, ellas trabajan alrededor de 10 a 12 horas más a la semana que los varones en el trabajo total (INEGI, 2013). Esto es reflejo de la doble jornada femenina, que se realiza tanto fuera como dentro del hogar.

En este caso, lo que queremos resaltar es que el trabajo remunerado es fundamental para la sobrevivencia de los hogares rurales, sin desconocer también que hay cuestiones simbólicas que están presentes en la motivación por el ingreso monetario. Sin embargo, es importante reconocer las diversas jornadas y roles de trabajo que re-

8. En ambos sexos, la población que tiene entre 30 y 59 años de edad es quien más horas labora a la semana en el trabajo total. 
quieren hacer las mujeres tanto en casa y fuera de ella, como es el trabajo agrícola, el cuidado de ancianos y niños, las tareas del funcionamiento del hogar, la participación comunitaria (i.e iglesia y fiestas patronales), entre otros, y que no son remunerados y son fundamentales para la reproducción del grupo doméstico. Así, en el caso de la mujer en el ámbito rural, cuando hablamos de pluriactividad no solo nos referimos a la extensión y desplazamiento a faenas remuneradas. La combinación entre las actividades agrícolas y no agrícolas tienden a ser más intensas en la medida que son más complejas y diversas las relaciones entre los agricultores y el medio ambiente social y económico en donde están ubicados. De ahí la importancia de recuperar las transformaciones espaciales y socioeconómicas.

Esta diversidad de actividades se genera en un mismo espacio (rural o periurbano), el que se diversifica y cambia de manera acelerada. En este sentido, la pluriactividad actual depende ahora de cómo se articulan los espacios rurales con las dinámicas asociadas a los nuevos usos de suelo ligados con el proceso de urbanización. Esto, sin duda, es fuente de una creciente diversidad de oportunidades y quehaceres entre los distintos miembros de las familias rurales.

Otro aspecto importante de reconocer es el papel de la familia. Gras (2004: 95) subraya que la pluriactividad es un atributo o cualidad de la familia, aun cuando su observación se realice en el nivel del individuo. La autora define como "familia pluriactiva" cuando por lo menos uno de sus miembros está ocupado o realiza actividades diferentes a la propia producción agrícola, pudiendo o no dedicarse también al trabajo en la unidad económica tradicional. Complementariamente, Schneider y Conterato (2006: 5-6) mencionan que aún cuando la determinación de emplearse es una cuestión personal, es en la familia donde se discuten y tejen las posibles alternativas laborales, ya que el objetivo último es la sobrevivencia y reproducción del grupo doméstico.

El municipio de Tepetitla de Lardizábal tiene una población total de 18,725 habitantes (INEGI, 2010); el $48 \%$ son hombres y el $52 \%$ son mujeres. Las principales localidades son Tepetitla, San Mateo Ayecac, Guadalupe Victoria y Villa Alta, con una población que se aprecia en la figura 2. 
Figura 2. Población total por localidad en el municipio de Tepetitla de Lardizábal

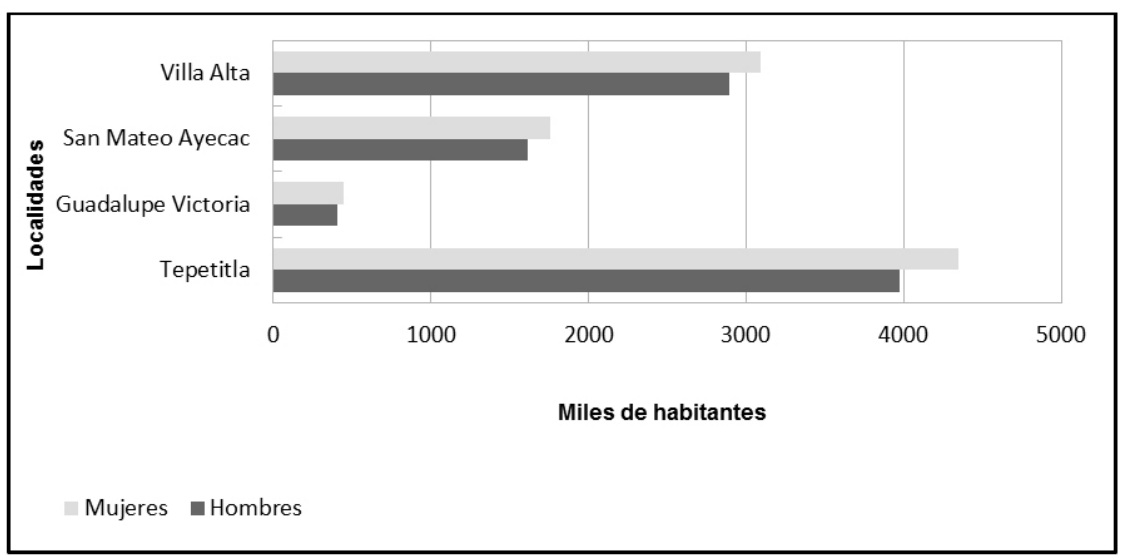

Como se muestra en la figura 2, existe un importante porcentaje, superior al 30\%, de la población económicamente activa femenina, siendo la localidad de Guadalupe Victoria la que registra mayor porcentaje de población económicamente activa (PEA) femenina con el $37.22 \%$.

Figura 3. Porcentaje de la población económicamente activa, Tepetitla de Lardizábal, 2010.

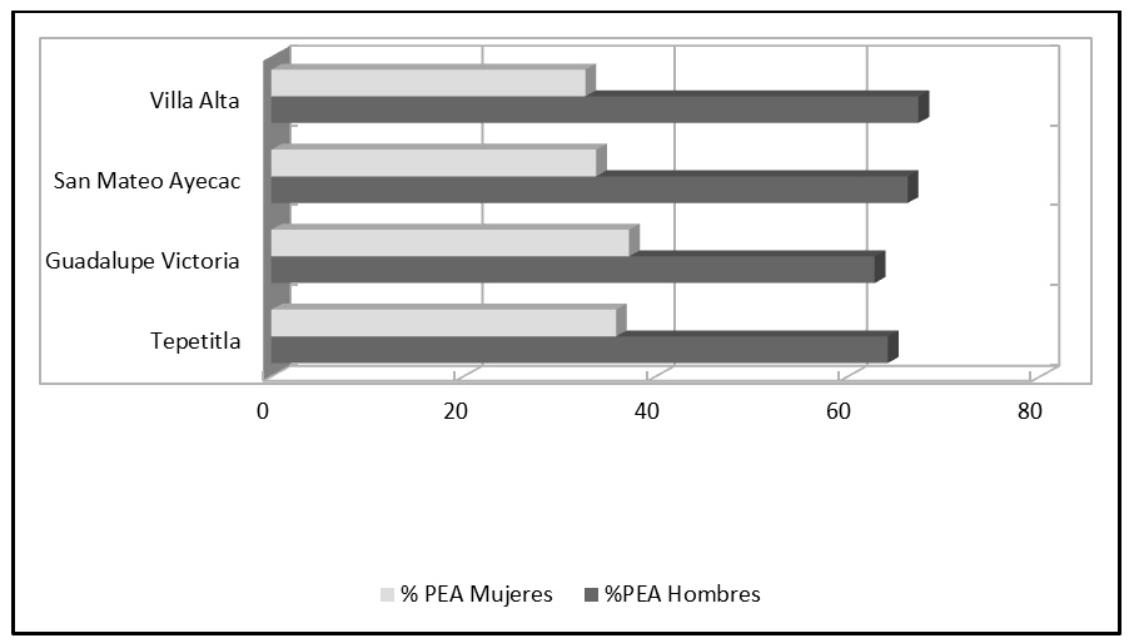

Se trata de localidades pequeñas todas. Y es este rasgo lo que resalta como importante por la profundidad de la transformación. Así, debemos decir que se bifurcan las lecturas sobre el cambio en la estructura social local, ya que por un lado se realza la inserción de las mujeres al mercado del trabajo, reconociendo el mundo femenino como uno de los sectores más favorecidos por ciertas ramas de la economía (De la 
$\mathrm{O}$, 2006), como ocurre con la industria textil y concretamente en las maquiladoras de confección. Por otro lado, lo que apostamos en este artículo, se apoya en la amplitud de la pluriactividad, donde el trabajo en la maquila es uno que se suma a los ya existentes como fuente de ingresos; principalmente, porque los "otros trabajos" no desaparecen, agudizándose a través del taller maquilador los procesos de explotación indirectos hacia otros miembros de la unidad familiar. Por eso, no desaparece completamente la actividad agrícola, aunque a veces lo parezca; ya que de acuerdo a los datos arrojados en el cuestionario, el $8 \%$ de la población de la muestra realiza actividades agrícolas. Es importante señalar que, realizando un análisis por grupos de edad y ocupación, la actividad agrícola se concentra en grupos de edad de más 45 años.

En cuanto a la pluriactividad, encontramos que en poco más del 53\% del Municipio, algún miembro reportó realizar entre dos y tres actividades para la subsistencia de la familia y cerca del $8 \%$ señaló realizar cuatro ocupaciones. Como indica una de las entrevistadas:

"En la costura así es, tienes que trabajar duro para sacar lo necesario, casi todos pagan por destajo (...) yo trabajo para varios patrones por así decir, busco corte de pantalón de mezclilla. Trabajo más en las mañanas cuando se va mi hijo a la escuela, aprovecho a darle a la costura, porque ya saliendo necesito ponerle atención (...) con la comida y limpieza me ayuda mi mamá, la lavada me toca a mí. Como mis papás ya son grandes también hay que atenderlos (...) Mi papá tiene poca tierra, poco menos de una hectárea, la mitad la da a medias y la otra la sembramos con maíz, en la cosecha me doy tiempo para desgranar y almacenar el maíz, como es de temporal no requiere tanto cuidado, aunque si hay que ver la siembra, la fertilizada, el deshierbe..." (Mercedes, comunicación personal, 12 febrero de 2016).

Entonces, es importante subrayar que uno de los aspectos por los cuales las mujeres buscan un trabajo remunerado, fuera o dentro del hogar, es para "complementar" la economía familiar dentro de un sistema de referencia de base agrícola. Sin embargo, para quienes son jefas de familia, el trabajo remunerado se constituye como la única fuente de ingresos. Para el caso de Tepetitla de Lardizábal, apoyado por el Censo de Población y Vivienda (2010) y en los propios instrumentos cuantitativos, se registran porcentajes superiores al $20 \%$ de hogares con jefatura femenina como apreciamos en la figura 4. 
Figura 4. Porcentaje de hogares de acuerdo al tipo de jefatura, Tepetitla de Lardizábal, 2010.

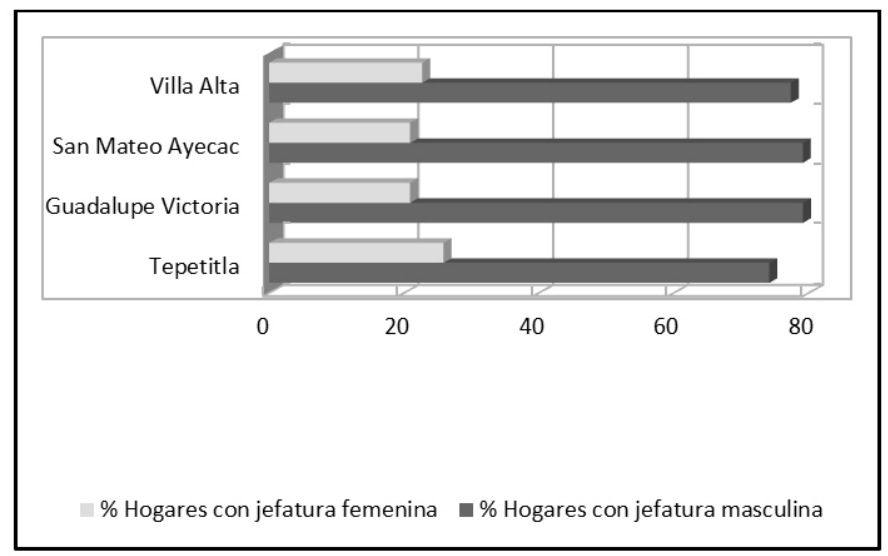

De acuerdo a las entrevistas realizadas en el municipio, el trabajo de las mujeres en los últimos años ha sido fundamental para el sostenimiento de la economía familiar, sobre todo por el aumento de desempleo, por la reducción real del salario y el debilitamiento de la producción agrícola. Como menciona una entrevistada: "cada vez el dinero alcanza para menos, entonces hay que entrarle al trabajo" (A. Norma, comunicación personal, 16 de febrero de 2015).

Las mujeres se emplean en los más diversos sectores, la industria textil y de la confección parece ser una alternativa más en donde pueden laborar. Sin embargo, este sector muestra aspectos interesantes en la conformación del mercado de trabajo, ya que da cuenta de la segmentación por sexo. Un ejemplo claro es el caso de las lavanderías, en donde contratan principalmente hombres, con un salario más alto. El argumento es que el trabajo realizado en las calderas requiere mayor fuerza física, horarios más extenso y/o nocturnos, que "no puede realizar una mujer". En estos espacios las mujeres son contratadas para las tareas menos calificadas como el planchado.

También es importante mencionar el tema de los tiempos de trabajo. En la mayoría de las lavanderías se trabaja continuamente durante las 24 horas, pues no se detienen las faenas. Como lo menciona un entrevistado: "día y noche se trabaja, el fuego de la caldera se tiene que mantener a cierta temperatura (A. Juan, comunicación personal, 4 de diciembre de 2014)". Dentro de un régimen que no deja de ser patriarcal, los horarios nocturnos son casi exclusivos para los varones, por lo que la posibilidad efectiva es que trabajen mujeres sólo en los turnos matutinos. El mismo entrevistado señaló: que además del esfuerzo físico, se tiene que considerar la rotación de turnos, y de acuerdo a su experiencia "para las mujeres es más difícil que les den permiso trabajar de noche (...) porque sus maridos no las dejan, o porque tienen que ver a sus hijos" (A. Juan, comunicación personal, 4 de diciembre de 2014). 
Los talleres de costuras son "la mejor alternativa" para las mujeres, ya que es ahí donde son más requeridas. Sin embargo, no es solo privativa de ellas, este nicho laboral cada día está más disputado por los varones, ya que de acuerdo a nuestra encuesta, para el año 2015, el 51\% de las ocupaciones en la confección eran desempeñadas por varones. La falta de empleo y el deterioro de actividades agrícolas han causado que un buen número de hombres se estén empleando en esta parte del proceso. Esto implica, paradójicamente, una mayor competencia en el mercado laboral para las mujeres, las que en ciertos talleres son desplazadas por los hombres bajo el argumento que "tienen más restricciones en los horarios y que si son madres los hijos las hacen faltar". A pesar de la presencia de los hombres en esta parte del proceso, aún existen actividades que están más feminizadas que otras, como son los terminados: deshilados, planchado, elaboración de ojal, elaboración de plantillas para aplicaciones. Sin embargo, estas actividades son las peores remuneradas en la cadena de especialización. Como señala una entrevistada: "el ojal te lo pagan a cincuenta centavos, hay plantillas sencillas que te ganas un peso y tardas en hacerla lo mismo que si cocieras unas cinco pretinas, es muy poco lo que pagan" (Olga, comunicación personal, febrero 2016).

Frente a lo anterior, son las mujeres las que enfrentan mayores desafíos laborales pese a la apertura de fuentes de trabajo. Por una parte, son excluidas de ciertas tareas, las cuales son mejor pagadas como aquellas que se desarrollan en las lavanderías; $y$, por otra, tienen que competir con los hombres que se emplean como costureros. A esto podemos añadir una menor flexibilidad en sus tiempos de trabajo fuera y dentro del hogar, ya que la carga de trabajo doméstico, la crianza de los niños, el cuidado de ancianos y enfermos sigue siendo tarea casi exclusiva de las mujeres.

El trabajo remunerado de las madres solteras, así como de mujeres solteras que aún viven con sus padres, es de tal importancia que pocas mujeres declararon sólo dedicarse al hogar. También es un hecho importante que buena parte de las trabajadoras casadas, cuyo esposo es considerado como el proveedor económico, continúan percibiendo la actividad laboral como una "ayuda complementaria", no reconociéndola como vital para su reproducción. Caso contrario al de las madres solteras, que sí reconocen que el trabajo de la mujer es importante no sólo dentro del hogar, sino que dinamiza la economía local:

"El trabajo de las mujeres es importante porque así circula más dinero, hay más movimiento, si sólo trabajaran los hombres, ¡imagínese!, ahora casi todas tienen que trabajar sean casadas o solteras, porque el dinero no alcanza, además porque si quieres salir adelante es más fácil si se apoya a la pareja, ya hay otro ingreso y no sólo está uno esperanzado en el gasto del señor. Aquí en San Mateo, las mujeres le entramos al trabajo, más a las que no nos gusta que nos manden... en el campo, en la costura, hasta en la lavandería, ahí estamos, aunque echemos pleito porque no es fácil dejar a los hijos, al marido, no es fácil" (A. Beatriz, comunicación personal, 31 de noviembre de 2014). 
La importancia del papel de las mujeres en el desarrollo económico local ha ido acompañada de una serie de contradicciones en ciertos sectores en los que las mujeres se emplean. Cierto es que las mujeres trabajadoras lo ven como una "victoria", y en cierto modo lo es, pero no en pocas ocasiones esta "victoria" está constituida por dificultades cotidianas difíciles de superar, ya que el trabajo de las mujeres -fuera del hogar- implica en la mayoría de veces "negociar sus derechos" con los otros miembros de la familia. Así, el lugar en donde se trabaja, con quién se emplea, la distancia de recorrido al trabajo, los horarios, entre otros aspectos, son puestos en la mesa de discusión cotidiana cuando una mujer decide ir por ingresos propios. Esto deja ver que, si bien la parte económica, es decir, la recepción de ingresos es importante para la sobrevivencia de la unidad doméstica, las negociaciones cotidianas en torno al empleo dejan ver problemas no resueltos sobre la equidad de género.

\section{Conclusiones}

Esta diversidad y complejidad en la esfera del trabajo y de las estrategias de sobrevivencia femenina sirven de entrada para entender la conformación de los mercados del trabajo en la interpenetración bidireccional de la urbe y la ruralidad, los cuales están siendo construidos y reconstruidos de una forma particular en el medio rural actual. De la misma forma nos permite comprender la manera en que los miembros de unidad doméstica se integran en distintas actividades económicas a redes de acumulación nacionales o globales, aún cuando no tengan conciencia de ello. Particularmente nos referimos a la explotación indirecta por parte del capital de los miembros más jóvenes que quedan al mando del hogar. Detrás de cada mujer empleada, en un taller sirviendo intereses externos, hay otros miembros de la unidad familiar que suplen su rol en la administración de algunas de las tareas del hogar, que no son remuneradas y que son "empleados indirectos" de las grandes empresas nacionales o transnacionales.

En muchos lugares, a partir de las reformas neoliberales de los años ochenta, se puede observar una mayor participación de las mujeres en el mercado del trabajo. Las crisis económicas y financieras, los planes de estabilización y las reformas económicas estructurales, incrementaron los niveles de pobreza en donde las mujeres resultaron ser las más afectadas. En muchos hogares las mujeres hoy pasan a convertirse en proveedoras de los ingresos familiares, ocupándose hasta en dos y tres jornadas laborales. Esto contradice la lectura autocomplaciente de las autoridades que solo ven las cifras de incorporación de las mujeres al empleo formal, pero no la precarización y la autoexplotación de la que son parte. La mayor de las veces, después de vender su fuerza de trabajo durante largas jornadas, regresan a ocuparse de las tareas del hogar y, si se requiere, en trabajo agrícola. En lo substantivo, son proveedoras de sus familias y también jefas de hogar, en una sociedad que, pese a los cambios descritos, prevalecen relaciones sin equidad de género. 
El mercado de trabajo descrito a través de este estudio de caso, es una muestra clara de las transformaciones socioterritoriales causadas por la globalización y su fase neoliberal, y cómo el desaparecimiento de la pequeña producción agrícola deja terreno fértil para las grandes corporaciones agroindustriales. La decantación de las mujeres al mercado del trabajo precarizado, tóxico y sin tiempo regulado en estos espacios, pone de manifiesto varias cuestiones: a) la necesidad de incorporación de más miembros del grupo doméstico en la búsqueda de ingresos, b) la multiplicación de las jornadas de trabajo para las mujeres, c) prácticas poco justas y equitativas hacia la mujer dentro y fuera del hogar con respecto al trabajo (remunerado y no remunerado), d) mayor tensión entre los miembros del hogar en el momento de la toma de decisiones respecto al trabajo fuera del hogar por parte de las mujeres, e) la nueva jerarquización y estratificación comunitaria entre aquellos que compran y los que venden fuerza de trabajo.

Varios hechos deben ser revelados en torno a la conformación de los actuales mercados de trabajo, ya que existe una clara presencia de trabajos más precarios en las zonas rurales, principalmente por la inexistencia de regulación laboral. Como hemos presentado en las páginas precedentes: cada vez más se requiere de ampliar jornadas de trabajo o multiplicar las mismas para obtener ingresos que permitan la sobrevivencia; existe en la base un empeoramiento del ingreso de los hogares, lo que da mayor peso en los hogares a nuevos roles para la satisfacción de necesidades; la violencia intrafamiliar es reforzada por las regresivas condiciones socioeconómicas. Estos aspectos, que pueden ser laterales y que esconden las cifras oficiales, sin duda obligan a tener una mirada crítica sobre el mundo del trabajo en general y de la participación de las mujeres en particular.

En los espacios rurales como el de Tepetitla de Lardizábal, aunque los ingresos propios perfilen subjetivamente la idea de cierta autonomía femenina, el análisis socioantropológico revela también procesos velados. Queda pendiente, entonces, la discusión sobre los innumerables retos sociales y políticos en torno al reconocimiento de la participación de las mujeres rurales en el desarrollo económico, el mundo del trabajo dentro del capitalismo, su flexibilidad, la vigencia de la doble explotación, así como de la promoción y defensa de sus derechos laborales.

\section{Referencias}

Alonso, Jorge (2002). Maquila domiciliaria y subcontratación en México en la era de la globalización neoliberal. México: Colegio de Tlaxcala, Plaza y Valdés.

Arias, Patricia (2013) "Migración, economía campesina y ciclo de desarrollo doméstico. Discusiones y estudios recientes”. Estudios Demográficos y Urbanos, 28 (1), 93-121. 
Arias, Patricia (2009). "Nueva estructura Ocupacional en el campo Latinoamericano" La pluriactividad en el campo latinoamericano. En Carton de Grammont, H. \& Martínez L. (coords.). Ecuador: FLACSO.

Beneria, Lourdes (2006). "La aparición de la economía feminista". Historia agraria, 17, 59-61.

Brenner, Neil (1999). "Globalization as reterritoriolization: re-scaling of urban governance in the European Union". Urban Estudies, 36 (3), 431-452.

Carton de Grammont, Hubert (2008). "El concepto de nueva ruralidad”. En Pérez, Edelmira; Farah, María y Carton de Grammont, Hubert (coord.) La nueva ruralidad en América Latina. Bogotá: FLACSO, Pontificia Universidad Javeriana.

De la O, María Eugenia (2006). “Geografía del trabajo femenino en las maquiladoras de México”. Papeles de Población, (49), 91-126.

Gobierno de Tlaxcala. (2014). Directorio Industrial. Disponible en http://www.contactopyme.gob.mx/parques/empresa.asp?ID=118

Gómez, Miguel Ángel (2004). "Reflexiones sobre el concepto de embbedness". POLIS, Investigación y Análisis Sociopolítico y Psicosocial, 2 (o2), 145-164.

Gras, Carla (2004). "Pluriactividad en el campo argentino: el caso de los productores del sur santafecino". Cuadernos de Desarrollo Rural, (51), 91-114.

Gudarrama, Rocio (2008). "Los significados del trabajo femenino en el mundo global. Propuesta para un debate desde el campo de la cultura y las identidades laborales". Estudios Sociológicos, XXVI, (77), 321-343.

Harvey, David (2014). Diecisiete contradicciones y el fin del capitalismo. Madrid: Traficantes de sueños.

Herrera, Fernando (2005). Vidas Itinerantes. México: UAM.

INEGI. (2010). Censo de Población y Vivienda. Principales resultados por localidad. México.

INEGI. (2013). Mujeres y hombres en México 2013, México.

Martínez, Estela, Mathew Lorenzen y Adriana Salas (2015). Reorganización del territorio y transformación socioespacial rural-urbana: sistema productivo, migración y segregación en Los Altos de Morelos. México: Editorial Bonilla Artigas-IISUNAM.

Méndez, Marlon Javier (2008). "La construcción de mixturas rural-urbanas: una lectura subjetivizante”. Cuadernos de Desarrollo Rural, 1 (54), 129-152.

Montiel, Oscar (2009). Trata de personas: padrotes, iniciación y modusoperandi. México: INMUJERES.

Rau, Victor Horacio (2006). "La sociología de los mercados laborales en los estudios sobre el empleo agrícola”. Gaceta Laboral, 12 (3), 357-385.

Robertson, Roland (2003). Glocalización: tiempo-espacio y homogeneidad-heterogeneidad. Cansancio del Leviatán: problemas políticos de la mundialización. Madrid: Trotta. 
Salas, Hernán y Leticia Rivermar (eds.) (2014). Nativitas, Tlaxcala: la construcción en el tiempo de un territorio rural. México: UNAM-IIA.

Sassen, Saskia (2015). Expulsiones. Buenos Aires, Mostoles- Madrid: Kattz Editores. Schneider, Sergio y Marcelo Antonio Conterato (2006). "Transformações agrarias, tipos de pluriatividade e desenvolvimento rural: considerações a partir do Brasil". En Neiman, G. \& Craviotti, C. (coord.) Entre el Campo y la Ciudad - Desafíos y estrategias de la pluriactividad en el agro. Buenos Aires: Ciccus.

Solow, Robert (1992). El mercado de trabajo como institución social. Madrid: Alianza. Velasco, Paola (2014). Antropología socioambiental. Ecología política, sujetos rurales y transformación del río Atoyac en el municipio de Nativitas, Tlaxcala (Tesis doctoral) recuperada de Tesis del Sistema Bibliotecario de la UNAM. Disponible en http://132.248.9.195/ptd2014/febrero/509004385/Index.html

Wolf, Eric (1971). Los campesinos. Barcelona: Editorial Labor.

Wolf, Eric (1977). Una tipología del campesinado Latinoamericano. Buenos Aires: Nueva visión.

\section{Sobre los autores}

Janett Vallejo Román es Profesora - Investigadora del Centro de Investigaciones y Estudios Superiores en Antropología Social -Unidad Golfo, México. Doctora en Geografía por la Universidad Nacional Autónoma de México, Maestra en Estudios Regionales por el Instituto de Invetigaciones Dr. José María Luis Mora y Licenciada en Sociología por la Universidad Autónoma Metropolitana-Iztapalapa. Correo electrónico es jvallejo@ciesas.edu.mx.

Juan Carlos Rodríguez Torrent es Profesor Titular e investigador en el Centro de Investigaciones en Vulnerabilidades e Informalidades Territoriales (CINVIT), de la Escuela de Diseño de la Facultad de Arquitectura de la Universidad de Valparaíso, Chile. Doctor en Ciencias Antropológicas por la Universidad Nacional Autónoma de México, posgraduado en la Facultad Latinoamericana de Ciencias Sociales (FLACSO) y licenciado en Filosofía y Antropólogo, titulado en la Universidad de Chile. Correo electrónico es juancarlosrodriguezt@yahoo.com. 


\title{
CUHSO. CULTURA-HOMBRE-SOCIEDAD
}

Fundada en 1984, la revista CUHSO es una de las publicaciones periódicas más antiguas en ciencias sociales y humanidades del sur de Chile. Con una periodicidad semestral, recibe todo el año trabajos inéditos de las distintas disciplinas de las ciencias sociales y las humanidades especializadas en el estudio y comprensión de la diversidad sociocultural, especialmente de las sociedades latinoamericanas y sus tensiones producto de la herencia colonial, la modernidad y la globalización. En este sentido, la revista valora tanto el rigor como la pluralidad teórica, epistemológica y metodológica de los trabajos.

\author{
EDITOR \\ Matthias Gloël \\ COORDINADORA EDITORIAL \\ Claudia Campos Letelier \\ CORRECTOR DE ESTILO Y DISEÑAdoR \\ Angélica Vera Sagredo \\ TRADUCTOR, CORRECTOR LENGUA INGLESA \\ Aurora Sambolin Santiago \\ Desarrollador de Sistemas \\ Laura Navarro Oliva \\ SITIO WEB \\ cuhso.uct.cl \\ E-MAIL \\ cuhso@uct.cl \\ LICENCIA DE ESTE ARTÍCULO
}

Creative Commons Atribución Compartir Igual 4.0 Internacional 